\title{
BMJ Open Benefits and barriers to participation in colorectal cancer screening: a protocol for a systematic review and synthesis of qualitative studies
}

\author{
Gladys N Honein-AbouHaidar, ${ }^{1,2}$ Monika Kastner, ${ }^{2,3}$ Vincent Vuong, ${ }^{1}$ \\ Laure Perrier, ${ }^{2}$ Linda Rabeneck, ${ }^{4,5,6,7}$ Jill Tinmouth,, ${ }^{3,6,7,8}$ Sharon Straus, ${ }^{2,3,7,9}$ \\ Nancy N Baxter ${ }^{1,2,3,6}$
}

To cite: HoneinAbouHaidar GN, Kastner M, Vuong $\mathrm{V}$, et al. Benefits and barriers to participation in colorectal cancer screening: a protocol for a systematic review and synthesis of qualitative studies. BMJ Open 2014;4: e004508. doi:10.1136/ bmjopen-2013-004508

- Prepublication history and additional material for this paper is available online. To view these files please visit the journal online (http://dx.doi.org/10.1136/ bmjopen-2013-004508).

Received 20 November 2013 Revised 7 February 2014 Accepted 10 February 2014

CrossMark

For numbered affiliations see end of article.

\section{Correspondence to}

\section{Dr Gladys N}

Honein-AbouHaidar; gladys.honein@mail.utoronto. ca

\section{ABSTRACT}

Introduction: Colorectal cancer (CRC) poses a serious health problem worldwide. While screening is effective in reducing $\mathrm{CRC}$ mortality, participation in screening tests is generally suboptimal and social inequities in participation are frequently reported. The goal of this review is to synthesise factors that influence an individual's decision to participate in CRC screening, and to explore how those factors vary by sex, ethnicity and socioeconomic status.

Data sources: A primary search of Cumulative Index to Nursing and Allied Health Literature (CINAHL), MEDLINE, EMBASE, PsycINFO, and a secondary search of grey literature and articles taken from references of included articles (from inception to July 2013).

Design: A systematic review and Meta-study synthesis of qualitative studies that address perceived benefits and barriers to participation in CRC screening tests among adults 50 years of age or older.

Review methods: The two-staged Meta-study methodology by Paterson will be used to conduct this review. In stage 1, similarities/differences, patterns and themes will be identified across three levels of analysis while preserving the context of original studies. In stage 2, synthesis will extend beyond the analysis to generate new theory of the phenomenon through a process called Meta-synthesis.

Discussion: This review offers to generate a framework to better understand benefits and barriers that affect decision-making to participate in CRC screening among different sectors of the population. This framework will be a relevant tool for policy makers in framing educational materials, for patientcentered communication, and for researchers interested in the science of equity. This review is registered in PROSPERO (registration number: CRD42013005025).

\section{INTRODUCTION}

Colorectal cancer (CRC) poses a serious health problem worldwide. CRC is the second

\section{Strengths and limitations of this study}

- This will be the first synthesis of qualitative studies to investigate why individuals undergo colorectal cancer (CRC) screening, their perceptions of and experiences with CRC screening and which aspects of screening are valued and culturally acceptable.

- The work will advance the science of conducting Meta-study reviews by rigorously executing its steps in the context of our research question and to document this process extensively in our final report.

- The work will advance the science of equity by identifying the determinants of social inequities in CRC screening participation.

- Findings from this Meta-study will be used to generate a framework to better understand the perceived benefits and barriers that affect individual decision-making of CRC screening.

- Findings may be limited to individuals from different ethnic minorities living in developed countries, which may limit the transferability of our findings to the overall ethnic population.

most common cause of cancer death in the USA, ${ }^{1}$ Canada, ${ }^{2}$ the UK, ${ }^{3}{ }^{4}$ Germany, ${ }^{5}$ Australia ${ }^{6}$ and Japan. ${ }^{7}$ It is estimated that by 2013, 142820 new CRC cases and 50830 CRC deaths will occur in the USA, ${ }^{1}$ and 23900 new CRC cases and 9200 CRC deaths will occur in Canada. $^{2}$

Screening for CRC can reduce the burden of the disease. Screening tests for CRC include faecal occult blood testing (guaiac FOBT) and faecal immunochemical test (FIT), flexible sigmoidoscopy, colonoscopy, CT colonography (CTC) and faecal DNA testing. Several of these tests are effective in reducing the incidence of, and in some instances, the mortality from the disease. Three landmark randomised controlled trials (RCTs) demonstrated that 
biennial use of guaiac FOBT coupled with colonoscopy in persons who tested positive was associated with a reduction in CRC mortality by $15 \% .^{8-10}$

Screening for CRC is a complex process, and many publicly funded healthcare systems have implemented an organised, population-based approach for screening such as in the UK, ${ }^{11}$ most provinces in Canada, ${ }^{12} 19$ of the 27 European Union (EU) countries, ${ }^{13}$ Japan $^{14}$ and Korea. ${ }^{15}$ Population-based organised screening programmes involve inviting a defined population at average risk for the disease (ie, people who do not have CRC, or strong family history of CRC, or medical conditions that put them at higher risk of developing CRC such as Crohn's disease or ulcerative colitis) to attend screening. The success of a high-quality organised, population-based CRC screening programme depends on adequate uptake as well as social equity in uptake. ${ }^{16}$ Early evaluation indicates an overall low participation and social inequity in participation. Participation in CRC screening tends to be lower among ethnic minorities, ${ }^{11}{ }^{17-19}$ low socioeconomic status individuals ${ }^{11} 20-22$ and among men. ${ }^{2022-24}$

While social inequities in uptake are well described in the literature, ${ }^{25}{ }^{26}$ what is missing is a clear understanding of why CRC screening is or is not appealing to individuals, aspects of screening that are valued and those that are culturally acceptable. Qualitative studies are important sources for this information. To date, a wide range of qualitative studies have elicited views on the perceived benefits and barriers to participation in screening from a range of ethnic and socioeconomic groups in various countries. The in-depth analyses in these studies reveal the complexity of social factors that affect an individual's decision to participate in screening. For example, studies have shown that difficulties in doing screening tests at home (ie, FOBT) and the perceived need for screening while having no symptoms of colorectal disease are the main barriers for participation across different population groups. ${ }^{27} 28$ In certain cultures, men perceive colonoscopy as embarrassing, invasive and an affront to their masculinity. ${ }^{22-24}$ 29-37 Women, in general, believe that their experience with other cancer screening tests such as mammography encourages them to do CRC screening, ${ }^{38}$ and because they often assume the role of caregiver in a family, they value the importance of self-care and early detection in order to prevent personal and family suffering. ${ }^{22}$ Less education, consistently equated with poorer health literacy skills, is often cited as the main barrier for CRC screening among low SES individuals. Poor health literacy is associated with reduced ability to 'obtain, process and understand health information, ${ }^{22}$ and the likelihood of engaging in preventive health behaviours such as CRC screening. ${ }^{39-41}$ Other reported factors influencing participation in CRC screening among certain ethnic populations include maintaining a positive energy (qi) and spirit (jing shen), as well as the belief that moderation of exercise and diet were enough to control the 'toxins' and prevent CRC. ${ }^{19}$
Systematic reviews of quantitative studies have focused on investigating the efficacy of CRC screening tests, ${ }^{42} 43$ the determinants of CRC screening participation ${ }^{25} 26$ and the effectiveness of interventions to increase screening participation. ${ }^{26} 44{ }^{45}$ However, no synthesis of qualitative studies exists to investigate why individuals undergo CRC screening or not, their perceptions of and experiences with CRC screening and which aspects of screening are valued and culturally acceptable. A well-designed synthesis of qualitative studies is needed to achieve a greater conceptual understanding of the perceived barriers and benefits associated with participation in CRC screening. This understanding is a necessary step to direct intervention designs to raise overall participation, reduce inequities in participation and eventually reduce mortality from CRC.

The Meta-study approach, a commonly used method to synthesise qualitative studies, was the most suitable approach to answer our research question. We considered other methods such as the Realist review (which seeks to understand what works for whom, under what circumstances and why) and Meta-ethnography (which aims to uncover a new theory to explain a range of findings), neither focuses on the experiences of people specifically nor considers the quality of included studies as part of the analysis.

The objectives of our study are to systematically review the literature for qualitative evidence that explores the factors that influence the decision of individuals aged 50 years or over at average risk for CRC to participate in CRC screening, and how those factors vary by sex, ethnicity and SES. Our secondary aim will be to generate a framework to better understand the perceived benefits and barriers that affect individual decision-making.

\section{METHODS}

\section{Synthesis methodology}

We will use the Meta-study methodology to conduct our review, which is a systematical, analytical and synthesis research method pioneered by Paterson et al. ${ }^{46} \mathrm{We}$ selected this methodology because it was the most suitable to answer our research question. Meta-study is a multifaceted, systematical knowledge synthesis method aimed at better understanding how people construct knowledge. ${ }^{47}$ In the context of our study, this is related to better understanding the determinants of CRC screening test participation. More specifically, it is an interpretive qualitative research approach in the constructivist paradigm (ie, the role of the investigator is to understand how people construct knowledge about the phenomenon under study). ${ }^{48}$ The aims of Meta-study are to 'analyse' and 'synthesise' what has been reported in the literature-these are considered distinct. Analysis involves identifying commonalities, differences, patterns and themes in a body of qualitative research (ie, what is typically done in a qualitative systematic review). Synthesis extends beyond analysis to identify 'truths' about the 
phenomenon under study by considering how the primary researchers interpreted the data (ie, Meta-data), the design and quality of studies (Meta-method) and the theoretical frameworks or perspectives used in these research reports (Meta-theory). To answer our research questions, we need to go beyond the 'analysis' of existing literature, as CRC screening is complex, and currently, it is unknown why people do or do not undergo CRC screening. We hypothesise that there may be underlying factors involved in an individual's perceptions and experiences well beyond CRC as a disease itself that influences their decision to undergo diagnostic testing (eg, cultural beliefs). Meta-study will allow us to extend beyond the typical 'analysis' phase because it considers the triangulation of the raw data (meta-data) and its quality (meta-method) as well as the theoretical underpinnings of this data (meta-theory). This level of 'synthesis' called 'Meta-synthesis' will lead to a new understanding of CRC and screening decisions (eg, colonoscopy) beyond what would be discovered in a qualitative systematical review (which tends to focus entirely on the primary research findings).

Figure 1 Flow of proposed Meta-study methods.
The proposed flow of our Meta-study methods is represented in figure 1 . Our Meta-study will be guided by the reporting standards as outlined in the ENTREQ criteria (Enhancing Transparency in Reporting the Synthesis of Qualitative Research). ${ }^{49}$ This is a 21-item checklist grouped into five main domains: introduction, methods and methodology, literature search and selection, appraisal and synthesis of findings. The protocol has been registered in the PROSPERO database (registration number: CRD42013005025, available at http:// www.crd.york.ac.uk/PROSPERO).

\section{Eligibility criteria}

We developed our eligibility criteria from our research questions. The review will use the following PICOS (Population, Intervention, Context, Outcomes and Study design) elements: (1) Population: Adults aged 50 years or over referred for CRC screening; exclusion criteria are studies investigating participants previously diagnosed with CRC; a hereditary, personal or family history of CRG (eg, Familial Adenomatous Polyposis (AFP) and hereditary non-polyposis CRC (HNPCC));

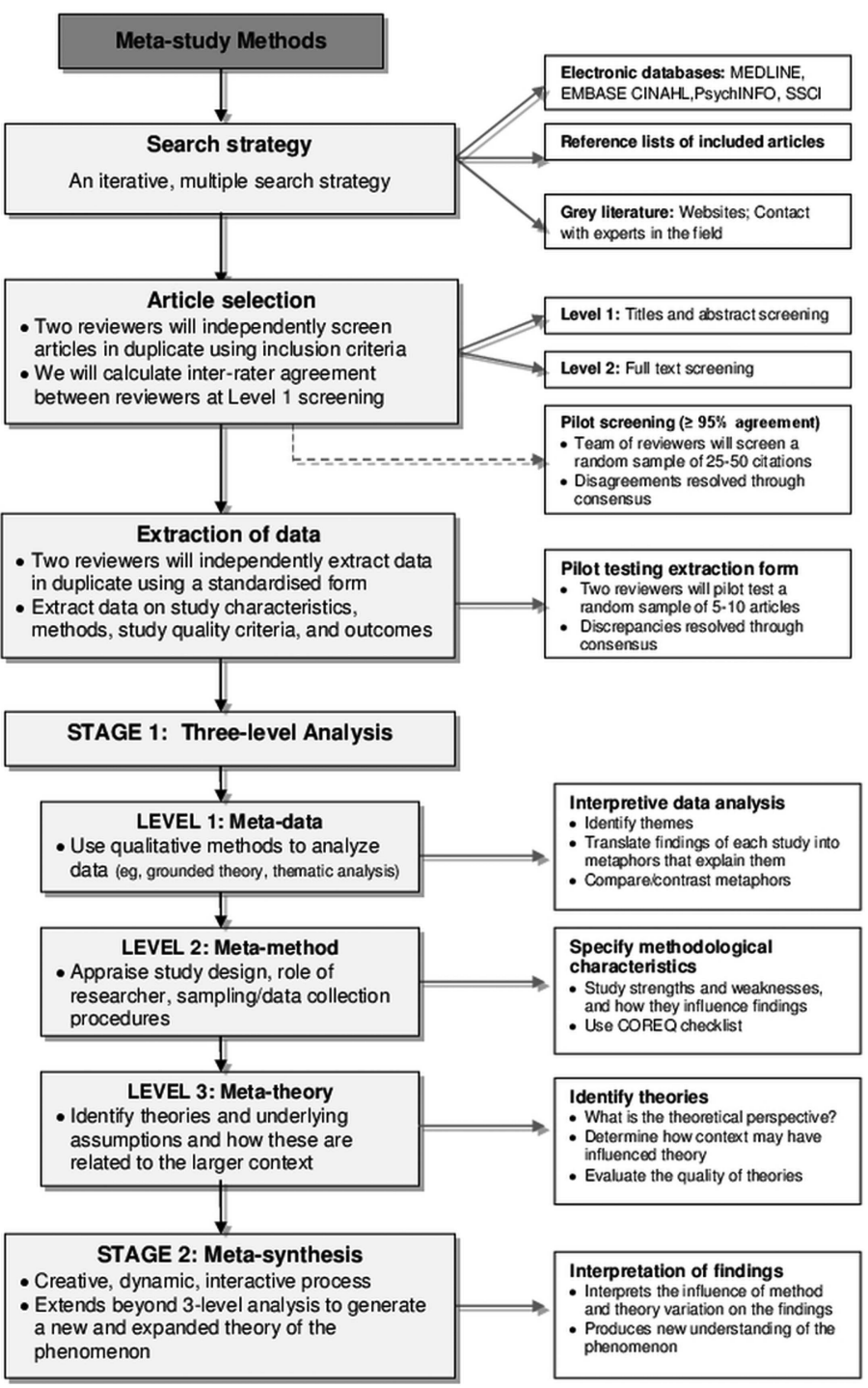


and a history of inflammatory bowel disease (eg, ulcerative colitis and Crohn's disease); (2) Intervention: We will identify all articles investigating perceptions of CRC screening as well as those investigating CRC as a disease ; (3) Context: We will investigate any variations in perceptions by sex, ethnicity, SES and other factors influencing CRC screening behaviour; (4) Outcomes: Perceptions related to CRC as a disease, causes of CRC, benefits and barriers to CRC screening and any other contextual factors that motivate or influence people's decision to participate in CRC screening; (5) Study design: We will include all qualitative studies and mixed-methods studies with a qualitative component. We will exclude experimental, observational and any non-empirical studies (ie, not based on observation or experience, opinion-driven or no hypothesis testing) such as editorials, letters, commentaries and narrative reviews.

\section{Information sources}

We will conduct a systematic search in the following electronic databases from inception to July 2013: MEDLINE, EMBASE, the Cumulative Index to Nursing and Allied Health Literature (CINAHL), PsycINFO and Social Science Abstracts (SSA). We will conduct a secondary search of the grey literature (unpublished) from sources such as Cancer Care Ontario and the National Health System Bowel Cancer Screening Programme. We will also search the reference lists of included articles and identify other articles through contact with experts in the field and linkages with our team members (eg, Cancer Care Ontario). There will be no language restrictions in our searches. We anticipate completing the review by April 2014.

\section{Search strategy}

Literature searching will be conducted by an experienced information specialist. The search strategy for the main database (MEDLINE) will be peer reviewed by another experienced information specialist using the PRESS checklist (ie, Peer Review of Electronic Search Strategies) ${ }^{50}$ The resulting retrieval yield will be limited to qualitative studies and mixed methods with a qualitative component using the optimised search strategy filter for qualitative studies of selected databases: MEDLINE, ${ }^{51}$ EMBASE, ${ }^{52}$ PsycINFO $^{53}$ and CINAHL. ${ }^{54}$ The draft search strategy for MEDLINE is available in online supplementary appendix 2. For the other databases, the search strategies are available from the authors on request.

\section{Study selection}

We will first perform a calibration exercise to ensure reliability of screening. Using the inclusion/exclusion criteria available in online supplementary appendix 1, two reviewers will independently screen a random sample of citations (25-50 citations) using our online Synthesi.SR Tool (proprietary online systematic review software developed for our Knowledge Synthesis Center at St. Michael's Hospital). ${ }^{55}$ We will calculate inter-rater agreement for study inclusion using per cent agreement, and repeat our pilot screening exercise until we reach at least $90 \%$ agreement at which point investigators will independently review titles and abstracts of potentially relevant articles in duplicate (level 1 screening). For level 2 screening, we will follow a similar calibration exercise as described for level 1 screening to identify full-text articles. Conflicts will be resolved through research team consensus for both levels of screening.

\section{Data collection process}

Two reviewers will abstract data independently using a standardised data collection form. The form will first be pilot tested on a random sample of 5-10 included studies and modified accordingly. Data abstraction will begin only if agreement is at least $95 \%$ among the two abstractors. We will extract data on study characteristics (eg, first author and citation) and qualitative study quality criteria according to the CASP tool (Critical Appraisal Skills Programme), which includes a 10-item checklist to assess the clarity of research aims, appropriateness of methodology and recruitment strategy, data collection, ethical considerations including the relationship between researcher and participants, the rigour of analysis, clear statement of findings and the value of the research. ${ }^{56}$ All data abstraction will be conducted using our online Synthesi.SR Tool, which provides a platform to resolve conflicts between reviewers directly in the system. Discrepancies will be reviewed and resolved by discussion among the team. The reporting of our review will be guided by the ENTREQ criteria. ${ }^{49}$

\section{Data synthesis}

We will perform a two-staged synthesis of the data (ie, Analysis and Synthesis) with the goal of creating a new interpretation of the phenomenon under investigation (ie, a new understanding of CRC and screening decisions; figure 1).

\section{Stage 1 (Analysis of data=Meta-data+Meta-method}

\section{+Meta-theory)}

We will identify the similarities and differences, patterns and themes across three levels of analysis. ${ }^{46} 4757$ Level 1 -Meta-data analysis: this will involve the interpretive analysis of research findings from primary studies to identify similarities and discrepancies among them using any one of several qualitative data analytical approaches (eg, line of argument; grounded theory; thematic analysis). The type of analysis method we select will be driven by the data that will emerge. In the context of our work, we anticipate that this will likely involve using thematical analysis to group themes (such as the benefits and barriers to CRC screening) according to sex, SES or other factors that emerge, and then noting the similarities and differences between them. Level 2 -Meta-method: This level of analysis will examine how the research methods and procedures in primary studies were used to generate and interpret data and shape the 
findings. It will include a process of appraising each included study according to the CASP tool for quality assessment of qualitative studies. ${ }^{56} \mathrm{~A}$ third reviewer will be available to settle discrepancies between reviewers for applying the CASP criteria. Level 3-Meta-theory: This level of analysis examines the theories that underpin the study authors' framing of their research questions, their criteria for inclusion and their conceptual framework for interpretation. It is the level at which the theoretical perspectives in qualitative reports can be interrogated to explain the phenomenon under study. We will review each report to identify the theoretical perspective used and the 'schools of thought' around CRC screening, and to determine how context may influence such perspectives.

\section{Stage 2 (Synthesis of data=Meta-synthesis)}

In stage 2, synthesis will extend beyond the three levels of analysis to generate a new and expanded theory of the phenomenon through a process called Metasynthesis. In contrast to the three-level analytic stage, Meta-synthesis is 'a creative, dynamic and interactive process that defies codification'. ${ }^{46}$ It involves interpreting the influence of method and theory variation in the findings to produce a new understanding of the phenomenon. For example, we will determine these influences by documenting how each study performs their data analysis (eg, thematic analysis of semistructured interviews=Meta-data analysis); whether they used a theoretical framework to drive their study (eg, the Health Belief Model=Meta-theory); and to determine the study quality (eg, the CASP criteria=Meta-method). Once we collect this data from all studies, we will be able to triangulate this data from individual studies to reveal a new, collective understanding of CRC screening participation. This interpretation will be documented during data extractions. To reduce the potential of bias introduced from such an interpretive process, two investigators will independently perform this interpretation, which will be discussed and finalised with input of the entire research team. We will use findings informed by the three-level analysis to develop a framework that shows the perceived benefits and barriers of CRC screening participation according to sex, SES, cultural beliefs and other factors that may emerge.

\section{Discussion and dissemination}

We will use findings from our in-depth analysis of qualitative studies to generate a framework to better understand the benefits and barriers that affect decisionmaking to participate in CRC screening among different sectors of the population. We anticipate that this framework will be relevant for a wide range of knowledge users: policy makers will be able to use the framework as a tool to frame educational materials to address barriers to CRC screening; and physicians may use it as a tool in patient-centered communication or in group education sessions in order to engage culturally heterogeneous population into a discussion on CRG screening. This review also offers advancement in the science of equity by identifying the determinants of social inequities in CRC screening participation. Using the anticipated framework, researchers may also design novel interventions to address those inequities, which may lead to improved quality in practice and advancement in evidence-based decision-making. Furthermore, synthesis of available qualitative evidence of barriers to participation in CRC screening currently does not exist. Therefore, our findings may trigger other systematic reviews of gaps in information that we may identify. We will also advance the knowledge of conducting Meta-study reviews by rigorously executing its steps in the context of our research question and to document this process extensively in our final report.

Our study may also have some limitations. As with any qualitative studies, our work may be susceptible to threats to internal validity (ie, credibility), external validity (ie, transferability) and reliability (dependability). ${ }^{57}$ We will address potential threats to credibility by pilot testing the data abstraction forms and involving group team discussions throughout the interpretation of findings. The knowledge produced in our review may not be transferable to other people or settings. For example, findings may be limited to individuals from different ethnic minorities living in developed countries, which may limit the transferability of our findings to the overall ethnic population. However, we will abstract a detailed account of the population and setting of each included qualitative study to maximise the potential for transferability of our findings. To limit the potential of biases that may be introduced by investigators with respect to the dependability and conformability of our work, we will standardise procedures, methods and analysis strategies across all aspects of the review process.

We will ensure broad dissemination of this synthesis review to include publication in open access journals as well as conference presentations. We will also plan to hold a meeting with our key stakeholders (ie, clinicians, researchers, people with CRC and decision-makers) to discuss the findings, to generate key messages most relevant to each and to discuss the next steps including the development of educational materials that will address gaps in CRC screening participation.

\section{Author affiliations}

${ }^{1}$ Department of Surgery, St. Michael's Hospital, Toronto, Ontario, Canada ${ }^{2} \mathrm{Li}$ Ka Shing Knowledge Institute, St. Michael's Hospital, Toronto, Ontario, Canada

${ }^{3}$ Institute of Health Policy, Management, and Evaluation, University of Toronto, Toronto, Ontario, Canada

${ }^{4}$ Dalla Lana School of Public Health, University of Toronto, Toronto, Ontario, Canada

${ }^{5}$ Vice-President, Prevention and Cancer Control, Cancer Care Ontario, Toronto, Ontario, Canada

${ }^{6}$ Institute for Clinical Evaluative Sciences, Toronto, Ontario, Canada

${ }^{7}$ Department of Medicine, University of Toronto, Toronto, Ontario, Canada

${ }^{8}$ Scientist at Sunnybrook Research Institute, Toronto, Ontario, Canada

${ }^{9}$ Department of Medicine, University of Calgary, Toronto, Ontario, Canada 
Contributors GHA and NB helped conceive the study; GHA, NB, MK and VV conceived the study design. GHA and MK helped draft the protocol. LP developed and executed the search strategy and edited the draft protocol. All authors helped editing the draft protocol, read and approved the final manuscript.

Funding This research was supported through a Cancer Care Ontario research grant and Canadian Cancer Society Research Institute award (grant number 2011-700803).

Competing interests NB holds the Cancer Care Ontario Health Services Research Chair.

Provenance and peer review Not commissioned; externally peer reviewed.

Data sharing statement Unpublished study data such as the search strategies for the other databases (EMBASE, CINAHL, PsycINFO, SSA) are available on request to the corresponding author.

Open Access This is an Open Access article distributed in accordance with the Creative Commons Attribution Non Commercial (CC BY-NC 3.0) license, which permits others to distribute, remix, adapt, build upon this work noncommercially, and license their derivative works on different terms, provided the original work is properly cited and the use is non-commercial. See: http:// creativecommons.org/licenses/by-nc/3.0/

\section{REFERENCES}

1. American Cancer Society. Cancer facts \& figures 2013. Atlanta: American Cancer Society, 2013

2. Canadian Cancer Society's Advisory Committee on Cancer Statistics. Canadian cancer statistics 2013. Toronto, ON: Canadian Cancer Society, 2013.

3. Cancer Research United Kingdom. Cancer mortality in the UK in 2010. Cancer Statistics for the UK, 2010 [14 Aug 2013]. http:// publications.cancerresearchuk.org/downloads/Product/CS CS MORTALITY.pdf

4. Cancer Research United Kingdom. Cancer incidence for common cancers. Cancer Statistics for the UK; [August 14, 2013]. http://www. cancerresearchuk.org/cancer-info/cancerstats/incidence/ commoncancers/\#Twenty

5. German Cancer Research Center in the Helmholtz Association. Leading causes of cancer deaths. Deutsches Krebsforschungszentrum, 2010. http://www.dkfz.de/en/krebsatlas/ total/mort 6 e.html

6. Australian Institute of Health and Welfare \& Australasion Association of Cancer Registries. Cancer in Australia: an overview, 2012. Canberra: AlHW, 2012

7. Foundation for Promotion of Cancer Research. Cancer statistics in Japan '12. National Cancer Center, 2012. http://ganjoho.jp/data/ professional/statistics/backnumber/2012/cancer_statistics_2012.pdf

8. Mandel JS, Bond JH, Church TR, et al. Reducing mortality from colorectal cancer by screening for fecal occult blood. Minnesota Colon Cancer Control Study. N Engl J Med 1993;328:1365-71.

9. Kronborg O, Fenger $\mathrm{C}$, Olsen $\mathrm{J}$, et al. Randomised study of screening for colorectal cancer with faecal-occult-blood test. Lancet 1996;348:1467-71

10. Hardcastle JD, Chamberlain JO, Robinson $\mathrm{MH}$, et al. Randomised controlled trial of faecal-occult-blood screening for colorectal cancer. Lancet 1996;348:1472-7.

11. Weller DP, Campbell C. Uptake in cancer screening programmes: a priority in cancer control. Br J Cancer 2009;101(Suppl 2):S55-9.

12. Cancer CPA. Approach to prevention. 2013 [cited 12 Nov 2013]. http://www.partnershipagainstcancer.ca/priorities/ prevention-screening/

13. Zavoral M, Suchanek S, Zavada F, et al. Colorectal cancer screening in Europe. World J Gastroenterol 2009;15:5907-15.

14. Saito $\mathrm{H}$. Colorectal cancer screening using immunochemical faecal occult blood testing in Japan. J Med Screen 2006;13(Suppl 1):S6-7.

15. Park MJ, Choi KS, Lee YK, et al. A comparison of qualitative and quantitative fecal immunochemical tests in the Korean national colorectal cancer screening program. Scand J Gastroenterol 2012;47:461-6.

16. Coombs A, Jones-McLean E, Le-Petit C, et al. Technical Report for the National Committee on Colorectal Cancer Screening. The National Committee on Colorectal Cancer Screening, 2002.

17. Javanparast S, Ward PR, Carter SM, et al. Barriers to and facilitators of colorectal cancer screening in different population subgroups in Adelaide, South Australia. Med J Aust 2012;196:521-3.
18. Severino G, Wilson C, Turnbull D, et al. Attitudes towards and beliefs about colorectal cancer and screening using the faecal occult blood test within the Italian-Australian community. Asian Pac $J$ Cancer Prev 2009;10:387-94.

19. Choe JH, Tu SP, Lim JM, et al. 'Heat in their intestine': colorectal cancer prevention beliefs among older Chinese Americans. Ethn Dis 2006;16:248-54.

20. Honein-Abouhaidar GN, Baxter NN, Moineddin R, et al. Trends and inequities in colorectal cancer screening participation in Ontario, Canada, 2005-2011. Cancer epidemiol 2013;37:946-56.

21. von Wagner C, Good A, Whitaker KL, et al. Psychosocial determinants of socioeconomic inequalities in cancer screening participation: a conceptual framework. Epidemiol Rev 2011;33:135-47.

22. Molina-Barcelo A, Salas Trejo D, Peiro-Perez R, et al. To participate or not? Giving voice to gender and socio-economic differences in colorectal cancer screening programmes. Eur J Cancer Care 2011:20:669-78.

23. Christy SM, Mosher CE, Rawl SM. Integrating men's health and masculinity theories to explain colorectal cancer screening behavior. Am J Mens Health 2013:8:54-65.

24. Getrich CM, Sussman AL, Helitzer DL, et al. Expressions of machismo in colorectal cancer screening among New Mexico Hispanic subpopulations. Qual Health Res 2012;22:546-59.

25. Beydoun HA, Beydoun MA. Predictors of colorectal cancer screening behaviors among average- risk older adults in the United States. Cancer Causes Control 2008;19:339-59.

26. Jepson $\mathrm{R}$, Clegg A, Forbes $\mathrm{C}$, et al. The determinants of screening uptake and interventions for increasing uptake: a systematic review. Health Technol Assess 2000;4:i-vii, 1-133.

27. Aubin-Auger I, Mercier A, Lebeau JP, et al. Obstacles to colorectal screening in general practice: a qualitative study of GPs and patients. Fam Pract 2011;28:670-6.

28. Brouse $\mathrm{CH}$, Basch $\mathrm{CE}$, Wolf RL, et al. Barriers to colorectal cancer screening with fecal occult blood testing in a predominantly minority urban population: a qualitative study. Am J Public Health 2003;93:1268-71.

29. Bass SB, Gordon TF, Ruzek SB, et al. Perceptions of colorectal cancer screening in urban African American clinic patients: differences by gender and screening status. $J$ Cancer Educ 2011;26:121-8.

30. Beeker C, Kraft JM, Southwell BG, et al. Colorectal cancer screening in older men and women: qualitative research findings and implications for intervention. $J$ Community Health 2000;25:263-78.

31. Fernandez ME, Wippold R, Torres-Vigil I, et al. Colorectal cancer screening among Latinos from U.S. cities along the Texas-Mexico border. Cancer Causes Control 2008;19:195-206.

32. Goldman RE, Diaz JA, Kim I. Perspectives of colorectal cancer risk and screening among Dominicans and Puerto Ricans: stigma and misperceptions. Qual Health Res 2009;19:1559-68.

33. Jones RM, Devers KJ, Kuzel AJ, et al. Patient-reported barriers to colorectal cancer screening: a mixed-methods analysis. Am j Prev Med 2010;38:508-16.

34. Thompson L, Reeder T, Abel G. I can't get my husband to go and have a colonoscopy: gender and screening for colorectal cancer. Health (London) 2012;16:235-49.

35. Wackerbarth SB, Peters JC, Haist SA. 'Do we really need all that equipment?': factors influencing colorectal cancer screening decisions. Qual Health Res 2005;15:539-54.

36. Winterich JA, Quandt SA, Grzywacz JG, et al. Masculinity and the body: how African American and White men experience cancer screening exams involving the rectum. Am J Mens Health 2009:3:300-9.

37. Winterich JA, Quandt SA, Grzywacz JG, et al. Men's knowledge and beliefs about colorectal cancer and 3 screenings: education, race, and screening status. Am J Health Behav 2011;35:525-34.

38. Chapple A, Ziebland S, Hewitson P, et al. What affects the uptake of screening for bowel cancer using a faecal occult blood test (FOBt): a qualitative study. Soc Sci Med 2008;66:2425-35.

39. Rakowski W, Meissner H, Vernon SW, et al. Correlates of repeat and recent mammography for women ages 45 to 75 in the 2002 to 2003 Health Information National Trends Survey (HINTS 2003). Cancer Epidemiol Biomarkers Prev [Research Support, N.I.H., Extramural]. 2006;15:2093-101.

40. Rutten LJ, Squiers L, Hesse B. Cancer-related information seeking: hints from the 2003 Health Information National Trends Survey (HINTS). J Health Commun 2006;11(Suppl 1):147-56.

41. Cairns CP, Viswanath K. Communication and colorectal cancer screening among the uninsured: data from the Health Information National Trends Survey (United States). Cancer Causes Control 2006; 17:1115-25. 
42. Hewitson $\mathrm{P}$, Glasziou $\mathrm{P}$, Watson $\mathrm{E}$, et al. Cochrane systematic review of colorectal cancer screening using the fecal occult blood test (hemoccult): an update. Am J Gastroenterol 2008;103:1541-9.

43. Whitlock EP, Lin JS, Liles E, et al. Screening for colorectal cancer: targeted, updated systematic review for the US Preventive Services Task Force. Ann Intern Med 2008;149:638-58.

44. Sabatino SA, Lawrence B, Elder R, et al. Effectiveness of interventions to increase screening for breast, cervical, and colorectal cancers: nine updated systematic reviews for the guide to community preventive services. Am J Prev Med 2012;43:97-118.

45. Brouwers MC, De Vito C, Bahirathan L, et al. Effective interventions to facilitate the uptake of breast, cervical and colorectal cancer screening: an implementation guideline. Implement Sci 2011;6:112.

46. Paterson BL, Canam C. Meta-study of qualitative health research: a practical guide to meta-analysis and meta-synthesis. SAGE Publications, 2001.

47. Paterson BL. The shifting perspectives model of chronic illness. J Nurs Scholarsh 2001;33:21-6.

48. Guba EG, Lincoln YS. Competing paradigms in qualitative research. In: INKDYSL, ed. Handbook of qualitative research. Thousand Oaks, CA: Sage, 1994:105-17.

49. Tong A, Flemming K, Mclnnes $\mathrm{E}$, et al. Enhancing transparency in reporting the synthesis of qualitative research: ENTREQ. BMC Med Res Methodol 2012;12:181.
50. Sampson M, McGowan J, Cogo E, et al. An evidence-based practice guideline for the peer review of electronic search strategies. $J$ Clin Epidemiol 2009;62:944-52.

51. Wong SS, Wilczynski NL, Haynes RB, et al. Developing optimal search strategies for detecting clinically relevant qualitative studies in MEDLINE. Stud Health Technol Inform 2004;107(Pt 1):311-16.

52. Walters LA, Wilczynski NL, Haynes RB, et al. Developing optimal search strategies for retrieving clinically relevant qualitative studies in EMBASE. Qual health res 2006;16:162-8.

53. McKibbon KA, Wilczynski NL, Haynes RB. Developing optimal search strategies for retrieving qualitative studies in PsycINFO. Eval Health Prof 2006;29:440-54.

54. Wilczynski NL, Marks S, Haynes RB. Search strategies for identifying qualitative studies in CINAHL. Qual Health Res 2007; 17:705-10.

55. The Joint Program in Knowledge Translation. Synthesi.sr Systematic Review Tool. St. Michael's Hospital. http://knowledgetranslation.ca/ sysrev/login.php

56. Critical Appraisal Skills Program (CASP) checklist for assessing qualitative studies. http://-www.caspuk.net/wp-content/uploads/2011/ 11/CASP-Qualitative-Research-Checklist.pdf (accessed Nov 2013).

57. Hansen HP, Draborg E, Kristensen FB. Exploring qualitative research synthesis: the role of patients' perspectives in health policy design and decision making. Patient 2011;4:143-52. 